\title{
2. Ausgangslage und Fragestellung
}

In den wirtschaftlichen Grossregionen der Schweiz wird das gesellschaftliche Leben zunehmend von internationalen Angestellten und ihren Familien mitgeprägt. Häufig wird für diese Personengruppe die Bezeichnung Expatriates (oder die Kurzform Expats) verwendet. Viele dieser - oftmals von ihren Arbeitgebern in die Schweiz entsendeten - Expatriates arbeiten in global agierenden Grossunternehmen mit internationalem Arbeitsumfeld (Müller-Jentsch 2008: 48ff); so ist auch tendenziell Englisch die vorherrschende Sprache am Arbeitsplatz. Zu dieser durch die Sprache geförderten „Campus Mentalität“ (Wiener/Grossmann 2011: 45) am Arbeitsplatz kommt hinzu, dass viele Expatriates in fremdsprachigen Netzwerken leben, in denen vor allem Englisch, Spanisch, Russisch, Chinesisch und Finnisch gesprochen wird. So lassen sich in der Schweiz bereits zahlreiche ,expat communities“(Von Arx 2008: 71) beobachten, in denen Expatriates sich in der jeweiligen Landessprache austauschen und vernetzen können. Zudem helfen firmeninterne ,welcome desks“ sowie ,relocation agencies“ bei den ersten Schritten in der neuen Heimat und kümmern sich um die Wohnungssuche, den Umzug, den Nachzug der Lebenspartnerin oder des Lebenspartners und - falls es Kinder gibt - um einen Platz an einer internationalen Schule. (Müller-Jentsch 2008: 58; Von Arx 2008: 75) Viele Expatriates bleiben somit eher unter sich in fremdsprachigen Netzwerken, separiert in der Arbeitswelt und mit eigenen Bildungseinrichtungen für ihre Kinder.

Eine Teilhabe am gesellschaftlichen Leben des Gastlandes Schweiz findet daher meist nur im begrenzten Rahmen der Unternehmen statt. Da dort die Arbeitssprache meist Englisch ist, werden Deutschkenntnisse nicht zwingend vorausgesetzt, was Austauschmöglichkeiten und Berührungspunkte mit der ansässigen Schweizer Wohnbevölkerung schmälert. Wird der Nachwuchs dazu noch auf eine internationale englischsprachige Schule geschickt, werden diese Tendenzen verstärkt. Zudem lassen sich auch Segregationstendenzen zwischen Expatriates und der Schweizer Bevölkerung beobachten. Die neuen Zuwanderinnen und Zuwanderer aus Ländern wie Grossbritannien, USA, Korea oder Japan konzentrieren sich bspw. im Wirtschaftsraum Zürich, in Zug oder in der Region Zimmerberg am Zürichsee. Sie haben vielfach die Tendenz, mit Gleichgesinnten der eigenen Sprach- und Kulturregion örtlich konzentrierte Communities zu bilden. (Stutz et al. 2010: 55) An dieser Stelle wird vielfach von sogenannten „Parallelgesellschaften“"gesprochen, in denen Expatriates leben. (Wiener/Grossmann 2011: 5; Von Arx 2008: 77)

Sprachbarrieren, die Isoliertheit am Arbeitsplatz und nicht zuletzt die zeitliche Begrenztheit des Aufenthaltes tragen somit auf der einen Seite dazu bei, dass sich für Expatriates meist eher wenige Austauschmöglichkeiten und Berührungspunkte mit der Schweizer Wohnbevölkerung ergeben. So bieten sich eher wenige Anreize, sich am gesellschaftlichen Leben des Gastlandes zu beteiligen oder sich zivilgesellschaftlich in irgendeiner Form zu engagieren. Auf der anderen Seite sind es aber auch die für Expatriates vielfach unbekannten gesellschaftlichen Regeln und Gepflogenheiten des Gastlandes Schweiz sowie die zum Teil vorkommende Verschlossenheit einheimischer Vereine und Organisationen, die diese Entwicklung beeinflussen. (Wiener/Grossmann 2011: 45) Es gelingt vielen Expatriates oftmals sogar nach langjährigem Aufenthalt nicht, in die Schweizer Gesellschaft „hineinzukommen“. (Staat 2011: 14)

(C) Der/die Autor(en) 2021

M. Störkle, Expatriates und freiwilliges Engagement in der

Schweiz, https://doi.org/10.1007/978-3-658-33043-9_2 
Einerseits kann festgehalten werden, dass dem Gastland Schweiz durch diese Entwicklung längerfristig ein grosses Potenzial an gesellschaftlichen Ressourcen von Hochqualifizierten entgeht. Für die schweizerische Zivilgesellschaft stellt diese Entwicklung eine verpasste Chance dar: Vereine, Institutionen und Organisationen könnten von diesem Potenzial profitieren. Gerade im Zusammenhang mit zugewanderten Führungskräften wird beispielsweise betont, dass eine lokale Verankerung sehr wichtig sei, damit eine gewisse Erdung vor Ort stattfinden könne. (Vögeli 2010: 18) Ansonsten bestehe hierbei die Gefahr, so die Befürchtungen, dass sich diese Führungskräfte gegenüber der einheimischen Belegschaft in keiner Weise verantwortlich fühlen würden. (ebd.: 18)

Andererseits sind Expatriates durchaus bereit, sich gesellschaftlich zu beteiligen, wobei sie dabei aber nicht nur angenehme Erfahrungen machen. Laut einer Studie zu den Expatriates im Raum Basel wären die darin Befragten zum Grossteil bereit, sich unter bestimmten Bedingungen gesellschaftlich in ihrer neuen Heimat zu engagieren, allerdings würden sie dabei häufig „mehr Widerstände als Ermutigungen“ (Wiener/Grossmann 2011: 6) erfahren. Gerade etwa im angelsächsischen Raum spielt die Freiwilligenarbeit eine wichtige Rolle im sozialen Leben. So waren viele Expatriates bereits in ihrer früheren Heimat, beispielsweise in Sportclubs, Vereinen oder Elternbeiräten organisiert. (Staat 2011: 14) Vielfach liegt bei diesen Expatriates zudem eine grosse Charity-Tradition vor, die allerdings anders funktioniert als beispielsweise das schweizerische Vereinswesen. (Wiener/Grossmann 2011: 52) Des Weiteren ist bei Expatriates die Tendenz beobachtbar, länger als geplant in der Schweiz zu bleiben, was die Wahrscheinlichkeit für ein mögliches und längerfristiges Engagement zusätzlich verstärken würde. (ebd.: 30)

Da auch zukünftig von einer wachsenden Zahl hochqualifizierter Zuwanderinnen und Zuwanderer in der Schweiz auszugehen ist (vgl. Migration-Mobility Survey 2018; Cattacin/Domenig 2012; Wiener/Grossmann 2011; Stutz et al. 2010; Baghdadi 2010; Müller-Jentsch/Avenir Suisse 2008), wird es zunehmend von Bedeutung sein, sich auch mit den gesellschaftlichen Ressourcen dieser Bevölkerungsgruppen zu befassen. Der Wissensstand zu den Ansprüchen, Ressourcen und Beteiligungsmöglichkeiten von Expatriates im Bereich des zivilgesellschaftlichen Engagements in der Schweiz, ist allerdings noch relativ gering. Es ist bisher noch nicht systematisch erforscht, ob die Schweiz für das Engagement von Expatriates überhaupt adäquate Beteiligungsmöglichkeiten bietet, resp. welche anderen Ansprüche dieser Bevölkerungsgruppe an zivilgesellschaftliche Beteiligungsformen stellt. Um sich diesem Forschungsgegenstand angemessen zu nähern, müssen allerdings beide Perspektiven in den Forschungsprozess miteinbezogen werden, die an Engagementprozessen beteiligt sind. So gilt es sowohl die Perspektive der Expatriates selbst als auch die Rahmenbedingungen in den Blick zu nehmen, unter denen Engagement entstehen kann und letztlich durchgeführt wird. Für die Forschungsanlage bedeutet das, sich beiden Seiten des Engagements aus einer Perspektive der Expatriates und einer Perspektive von Vereinen vor Ort zu nähern.

In der vorliegenden Studie möchte ich einen Beitrag zur systematischen Erarbeitung dieser Grundlagen liefern und ein Beitrag zur Schliessung dieser Forschungslücke im Bereich der qualitativen Forschungen zum freiwilligen Engagement leisten. Dabei nimmt meine Arbeit Bezug auf unterschiedliche Forschungsfelder, die zur theoretischen Rahmung meiner Forschung 
dienen. Die Arbeit siedelt sich zum einen in den Forschungen der (Freiwilligen-) Engagementforschung bzw. den Forschungen zur Zivilgesellschaft an (vgl. Freitag et al. 2016; Freitag 2014; Cattacin/Domenig 2012; Samochowiec et al. 2018; Kubisch/Störkle 2018; 2016), aus welchen sich auch letztlich der Forschungsbedarf ableiten lässt (vgl. Kap. 5). Darüber hinaus habe ich Forschungsansätze beigezogen, die sich mit den Lebenswelten von Expatriates beschäftigen. (vgl. Fechter 2007; Scott 2004; Nowicka 2006a; Cohen 1977) Die theoretischen Ansätze der transnationalen Migration (Dahinden 2009a; Faist et al. 2014; Pries 2008) werden dabei ebenfalls eine Rolle spielen, um die internationalen Biografien und transnationalen Aktivitäten besser zu verstehen, die bei vielen Expatriates zu finden sind.

\subsection{Expatriates im Kanton Zug - eine klassische Expatriate Destination}

Die vorliegende Arbeit ist als eine qualitative Fallstudie im Kanton Zug konzipiert, in dem das gesellschaftliche Leben in den letzten beiden Jahrzehnten stark von Expatriates geprägt wurde. Der Kanton Zug hat sich in den letzten Jahren zu einer klassischen „Expat-Destination“ entwickelt. (Marty 2010: 34) Als Teil des Wirtschaftsgrossraums Zürich ist der Kanton Zug ein wichtiger internationaler Handels-, Finanz- und Wirtschaftsstandort der Schweiz. Als einer der finanzstärksten Kantone der Schweiz, lockt Zug mit günstigen Steuern sowie der guten Erreichbarkeit und Nähe zu Zürich viele internationale Firmen mit ihren Angestellten an. (vgl. Merki 2011) Der Zuzug von Personen aus Ländern der sogenannten ,neuen Zuwanderung“"1 (Stutz et al. 2010: 7ff.) hat dort in den letzten fünfzehn Jahren stark zugenommen, sodass der Alltag in der Stadt Zug und anderen Zuger Gemeinden stark von dieser neuen Bewohnerschaft mitgeprägt wird. (ebd.: 49) So sind es die ausländischen und nicht die Schweizer Zuzüge, die seit einiger Zeit im Kanton Zug für das positive Bevölkerungswachstum verantwortlich sind. (Neff 2011: 23f.) Eine weitere Auswirkung der starken Zuwanderung sind die teuren Wohn- und Lebenshaltungskosten in Zug, die sich immer weniger ,ursprüngliche“ Zugerinnen und Zuger leisten können. Der teure Wohnraum bleibt vermehrt nur noch für die finanzstarken „,neuen“ Zuwanderinnen und Zuwanderer erschwinglich. Schaffner (2009) spricht im Tagesanzeiger in diesem Zusammenhang bereits vom „Exodus der Zuger“. Oft werden die Wohnkosten der Expatriates sogar von den Firmen übernommen, sodass eine zusätzliche Entfremdung zu den hohen Wohnraumkosten entsteht. Während man in wirtschaftsfreundlichen Kreisen den Zustrom der zahlungskräftigen Zuwanderinnen und Zuwanderer durchweg begrüsst und fördert, machen kritische Stimmen den wachsenden Anteil der Expatriates mitverantwortlich für die Entwicklung der steigenden Wohn- und Lebenshaltungskosten in Zug. (Marty 2010: 34)

Auch in anderen Lebensbereichen schlägt sich die neue Zuwanderung im Kanton Zug deutlich nieder. So gibt es neben internationalen Schulen und fremdsprachigen Kindergärten auch Expatriate-Netzwerke und Clubs wie den „International Men's Club of Zug“ (IMCZ), den „Zug

\footnotetext{
${ }^{1}$ Die ,neue Zuwanderung“ der Schweiz charakterisiert sich v.a. durch eine starke Zunahme hochqualifizierter Ausländerinnen und Ausländer vornehmlich aus den nördlichen und westlichen Ländern und durch einen Rückgang von Niedrigqualifizierten (v.a. aus den südlichen Ländern). (Stutz et al. 2010: 7ff.; vgl. hierzu auch MüllerJentsch/Avenir Suisse 2008; Baghdadi 2010; Piguet 2006; Löpfe/Vontobel 2011)
} 
International Women's Club“ (ZIWC) und das „Zug International Business Forum“ sowie zahlreiche Relocation-Agenturen, die bei der Wohnungssuche, dem Umzug, den ersten Behördengängen, der Handwerkervermittlung bis hin zur Einschulung schulpflichtiger Kinder in eine International School helfen. Die Website www.zug4you.ch richtet sich explizit an die ,growing Expat and international community“ in Zug und gibt lebenspraktische Informationen in englischer Sprache. Als Ergänzung zu dieser Website gibt es noch die Zug Post (www.thezugpost.ch), die in Zusammenarbeit mit der Zuger Zeitung täglich englischsprachige Nachrichten aus der Region veröffentlicht. Auf der Website des Kantons Zug findet man zudem englischsprachige Informations- und Merkblätter zu Behördengängen und weiteren Lebensbereichen, und die Kontaktstelle Wirtschaft des Kantons gibt einen englischsprachigen „Expat Guide“ für die neu zugezogenen Expatriates im Kanton heraus. (Marty 2010: 34ff.; vgl. Kanton Zug 2019) So finden sich im besagten „Expat Guide“ neben lebenspraktischen Hinweisen zu den Themen „moving here“, „,setttling in“, „everyday life“ und „leisure“ auch wichtige Kontakte zu Behörden sowie einführende Worte zur Schweiz, zum Kanton Zug und den typischen „social customs“ in der Schweiz. (Kanton Zug 2019: 5)

Während kritische Stimmen, so erörtert es bspw. Schaffner (2009) in einem Artikel, gegenüber diesen Entwicklungen bereits von einer deutlich erkennbaren sozialen Sonderbehandlung und Privilegierung sprechen, wodurch eine wachsende Zahl fremdsprachiger Expatriates in Parallelwelten leben und so wenig Anreize und Angebote haben, sich am gesellschaftlichen Leben der alteingesessenen Zuger Bevölkerung zu beteiligen, sprechen andere Stimmen von einer Bereicherung, die sich u. a. darin äussere, dass Zug ein zunehmend internationales Flair bekomme, oder eben Vereine wie etwa der ,Zug International Women“s Club“ überhaupt existieren. (ebd.) Die „Neuzugerinnen und Neuzuger“ geben nach Einschätzungen des einstigen Präsidenten des „International Men's Club of Zug“ allerdings ein sehr heterogenes Bild ab: „Den typischen Expat gibt es nicht. Zu verschieden sind Sprachen, Kultur, Pläne und Lebensumstände“, so wird der Präsident in einem Artikel von Marty (2010: 34) über die „Expatriate Welt“ im Kanton Zug zitiert. Expatriates, die für eine längere Zeit in Zug bleiben, würden allerdings durchaus Bereitschaft zeigen, sich am gesellschaftlichen Leben zu beteiligen. Bei den „short timers“, also Expatriates, die nur für kurze Zeit in Zug bleiben, gebe es allerdings vermehrt die Tendenz, in Parallelwelten zu leben. (ebd.: 34)

Die kurz skizzierten Gegebenheiten des Kantons Zug als „klassische Expat-Destination“ bilden eine ideale und spannende Ausgangslage, um mein Forschungsvorhaben zu den Themen Expatriates und Engagement zu realisieren. So ist es aber nicht nur die Aktualität der ExpatriatesThematik in Zug, die den Kanton spannend für meine Forschung macht. Es ist darüber hinaus der Aspekt, dass die oben skizzierte Entwicklung zu einer „Expat-Destination“ in einem vergleichsweise kleinen Kanton der Schweiz passiert, mit kleineren Orten wie Cham, Steinhausen und Baar, bis hin zu dörflich geprägten Orten wie Oberägeri und einer mittelgrossen Stadt Zug (mit rund 30'000 Einwohnerinnen und Einwohnern) als Hauptort im Zentrum der Entwicklung. Betrachten wir die Forschungsliteratur zu Lebenswelten von Expatriates, werden diese zu einem sehr grossen Anteil in den Metropolen dieser Erde lokalisiert, um mit Studien zu Expatriates in Singapur, New York, Jakarta, Paris oder London nur die Gängigsten zu nennen. (vgl. z. B. Beaverstock 2002, 2005; Scott 2004; Shimoda 2015) Für die Schweiz konstatieren 
die vorhandenen Studien eine räumliche Konzentration dieser neuen Zugewanderten vor allem in den Wirtschaftsräumen Basel, Zürich und dem Genfer Seebogen zwischen Genf und Lausanne. (Atmani 2009: 30; Stutz et al. 2010: 55; vgl. Wiener/Grossmann 2011) Der Kanton Zug liegt in unmitttelbarer Nähe zu Zürich und kann zur wirtschaftlichen Grossregion Zürich gezählt werden. Als bedeutsamer internationaler Handels-, Finanz- und Wirtschaftsstandort der Schweiz sticht der Kanton mit der betont wirtschaftsfreundlichen Ausrichtung besonders hervor. In der Selbstdarstellung des Kantons Zug im bereits erwähnten „Expat Guide“ heisst es bspw. in der Einleitung dazu:

The Canton of Zug is one of the most attractive business and residential regions in Switzerland. Despite its relatively small size, people from more than 137 nations live and work in our canton. They feel comfortable, particularly because commuting to work in Zug, reaching canton officials, and simply getting to the countryside from the city, are easy and convenient; additionally, they enjoy a rather diverse club and cultural life here.

The population of Zug is open to everything that's new and regards the constituency of foreigners here as enriching. Zug offers an attractive working environment because a large number of internationally networked companies provide employment opportunities for people of every level of education. Life in the Canton of Zug is multi-faceted thanks to an intact environment, a huge variety of leisure time opportunities and many cultural facilities. (Kanton Zug 2019: 3)

Diese betont Expatriate-freundliche Umgebung zeigt sich in Zug allerdings nicht nur auf der "Angebotsseite" des Kantons. Auch bei den Expatriates selbst scheint der Standort Zug sehr beliebt zu sein. In der im Jahr 2018 von InterNations, einem der weltweit grössten ExpatriateNetzwerke durchgeführten „Expat City Ranking“-Studie (2018a), erlangt die Stadt Zug im internationalen Beliebtheits-Ranking bei Expatriates aus 72 Städten den Platz 23, gleich hinter der Stadt Basel, die es ebenfalls als Schweizer Stadt ins obere Drittel auf den Platz 22 schafft. Die anderen Schweizer Städte landen bei den dort lebenden Expatriates eher auf den hinteren Plätzen, wie etwa Lausanne (Platz 44), Genf (Platz 56), Zürich (Platz 57) und Bern (Platz 61). Zum Vergleich: Auf die Top 10 Plätze dieses Rankings schaffen es im Jahr 2018 die Städte Taipeh, Singapur, Manama, Ho-Chi-Minh-Stadt, Bangkok, Kuala Lumpur, Aachen, Prag, Madrid und Maskat. Das „Expat City Ranking“ (InterNations 2018a) ist Teil einer seit 2014 jährlich durchgeführten „Expat Insider“-Studie (InterNations 2018b). Im Jahr 2018 konnte die Studie mit über 18'000 teilnehmenden Expatriates in 187 Ländern durchgeführt werden. Sie gibt dabei nicht nur einen Einblick in das Leben von Expatriates in 72 ausgewählten Städten auf der ganzen Welt, die Studie beinhaltet ebenfalls ein Ranking, das auf Basis von bestimmten Bewertungsfaktoren wie bspw. Eingewöhnung im Ausland, Arbeitsleben, Wohnen, Finanzen oder Lebensqualität erstellt wird. (ebd.)

Die Stadt Zug, die es in dieser Studie - wie bereits erwähnt - im weltweiten Vergleich insgesamt auf den Platz 23 bringt, sticht bei einigen Bewertungsfaktoren durch Spitzenplätze allerdings besonders hervor. So erhält die Kantonshauptstadt von den Expatriates weltweit die beste Bewertung der wirtschaftlichen Lage vor Ort. Ebenfalls weltweit an erster Stelle steht die Stadt Zug, wenn es um die Bewertung von Sicherheit und Politik sowie Umweltqualität geht. Im Vergleich mit allen anderen Schweizer Städten in der Studie (also Basel, Zürich, Genf, Lausanne und Bern) wird die Lebensqualität in Zug von den Expatriates am besten bewertet. Beim weltweiten Ranking der Lebensqualität kommt die Stadt Zug immer noch unter die ersten 
zehn (Platz 8). Auch das urbane Arbeitsleben schneidet bei den Expatriates in der Kantonshauptstadt im Schweizer Vergleich am besten ab, international liegt Zug hierbei auf dem 15. Platz. Bei anderen Bewertungsfaktoren schneidet die Stadt Zug hingegen weniger gut ab. So wird die Lage bei den örtlichen Lebenshaltungskosten in Zug am schlechtesten eingeschätzt (Platz 62 im internationalen Vergleich), Zürich (Platz 66) und Lausanne (Platz 69) werden von den Expatriates diesbezüglich allerdings noch schlechter bewertet. Generell empfinden die meisten der befragten Expatriates in Zug die Lebenshaltungskosten sowie das Wohnen in der Stadt Zug als zu teuer (jeweils 70 \%). Dem gegenüber stehen allerdings die Einschätzungen, dass rund 70 Prozent der befragten Expatriates in Zug angeben, mit ihrer finanziellen Lage zufrieden zu sein; nahezu alle Befragten ( $93 \%$ ) geben zudem an, dass sie ihr verfügbares Haushaltseinkommen für ausreichend bzw. mehr als ausreichend einschätzen. (Presseportal Schweiz 2018; vgl. InterNations 2018a)

Obwohl solche Rankings aus methodischer Sicht immer etwas mit Vorsicht zu geniessen sind, geben sie dennoch ein gewisses Stimmungsbild der dort lebenden Expatriates ab. Ähnliche charakteristische Merkmale lassen sich bspw. auf Expatriate-Plattformen in Zug feststellen. Auf der bereits erwähnten Website Zug4you werden die Qualitäten des Standorts Zug aus der Perspektive „von Expatriates für Expatriates“ wie folgt dargestellt:

Zug is a very beautiful canton in central Switzerland. The Canton boasts an ideal combination of a low tax rate, a well-educated population, and rapid access to other major Swiss cities. This makes Zug a small but very international canton. Major leading companies have chosen Zug as their Headquarters. The expat community is therefore quite a large one. (...)

The expat community particularly appreciates Zug's central location, with countries such as Germany, Austria, Italy and France all less than three hours away. The stable political situation, excellent public transport and its proximity to Lucerne and Zurich are also the reason why many expatriates have chosen Zug to become their new home. (Zug4you, ohne Datum)

Betrachten wir zuletzt noch die relevanten statistischen Kennziffern, um die dargelegte Ausgangslage im Kanton Zug noch mit einigen offiziellen Bevölkerungszahlen zu vervollständigen. In diesem Zusammenhang sind v. a. die Bevölkerungszusammensetzung und die Bevölkerungsentwicklung von Bedeutung. Der Kanton Zug hat in den letzten 20 Jahren einen deutlich positiven Bevölkerungssaldo vorzuweisen. Seit der Jahrtausendwende hat der Kanton Zug insgesamt eine Zunahme von 29'079 Personen zu verzeichnen, was einer Zunahme von 29,7 Prozent der Gesamtbevölkerung entspricht. Absolut wuchsen die beiden grössten Städte Zug (plus 8'079 Personen) und Baar (plus 5'717) am meisten, prozentual am meisten Zuwachs wies die Zuger Gemeinde Risch mit einem Plus von 56,3 Prozent auf.

Die Abbildung 1 zeigt diese Entwicklung noch einmal für alle Zuger Gemeinden auf: 


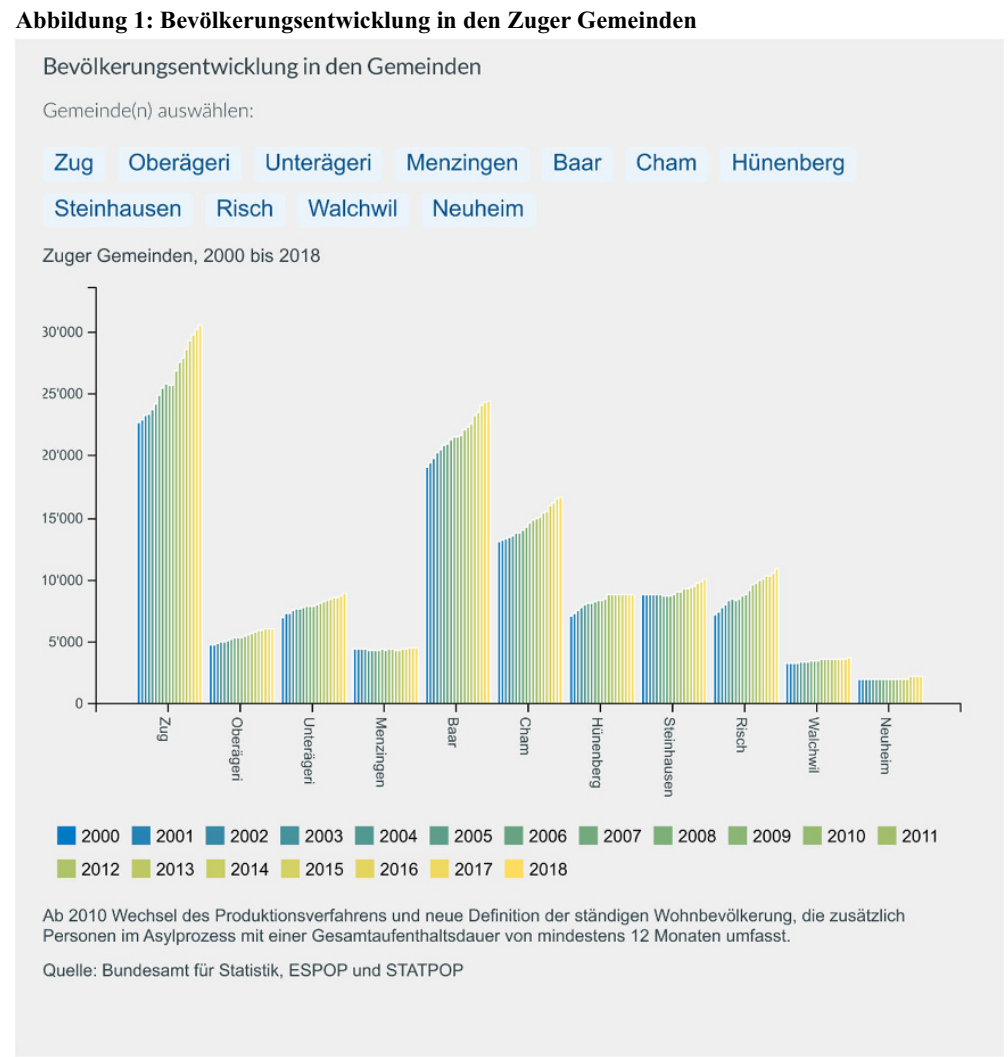

Hervorzuheben ist an dieser Stelle, dass v. a. die internationale Zuwanderung nach Zug in den letzten Jahren den Hauptgrund für diese Bevölkerungszunahme darstellt. Im Jahr 2018 sind insgesamt 2'967 Personen aus dem Ausland in den Kanton gezogen, in die Gegenrichtung haben ihn 2'165 Personen wieder verlassen. Der Kanton Zug verfügt im Ende des Jahres 2018 über einen Ausländer*innenanteil von 28,3 Prozent, was in absoluten Zahlen 35'920 Personen bedeutet. Im Vergleich zum Vorjahr 2017 ist dieser Anteil in Zug dabei geringfügig um 0,4 Prozentpunkte angestiegen. Anteilsmässig lebten im Jahr 2018 die meisten Ausländerinnen und Ausländer in der Gemeinde Walchwil (35,1 \%), die wenigsten in Hünenberg (17,6 \%). Diese Anteile sind, wie bereits erwähnt, in den letzten Jahren kontinuierlich angestiegen. Die nachfolgende Abbildung 2 zeigt diese Entwicklung im Kanton Zug seit dem Jahr 1990. Insbesondere sind dabei die roten Balken interessant, welche die Anteile der ausländischen Wohnbevölkerung in Zug darstellen. 


\section{Abbildung 2: Entwicklung der ausländischen Wohnbevölkerung im Kanton Zug}

\section{Ausländische Wohnbevölkerung}

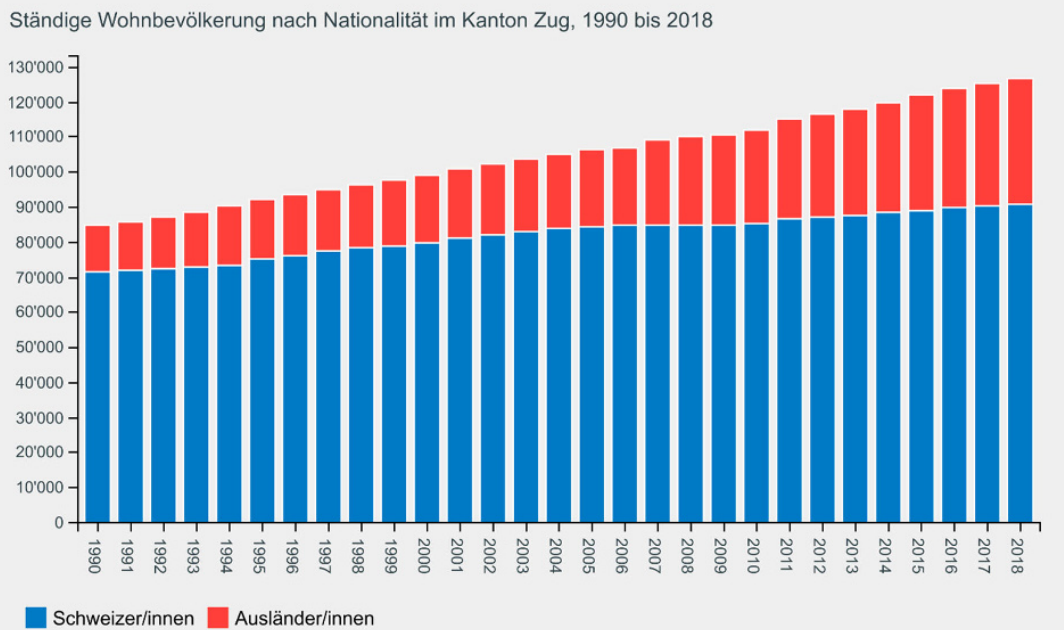

Ab 2010 Wechsel des Produktionsverfahrens und neue Definition der ständigen Wohnbevölkerung, die zusätzlich Personen im Asylprozess mit einer Gesamtaufenthaltsdauer von mindestens 12 Monaten umfasst.

Quelle: Bundesamt für Statistik, ESPOP (bis 2009) und STATPOP (ab 2010)

Der Anteil der Ausländerinnen und Ausländer an sich sagt an dieser Stelle jedoch noch nicht sehr viel über die Herkunft der Zugewanderten aus, da für diesen Anteilswert statistisch lediglich unterschieden wird, ob jemand den Schweizer Pass hat oder nicht. Betrachten wir noch genauer, welche Staatsangehörigkeiten die Zugewanderten im Kanton haben und wie sich ihre Anteile in den letzten Jahren verändert haben (siehe die Abbildungen 3 und 4).

Unter den ersten zehn am stärksten in Zug vertretenen Nationalitäten finden sich Ende 2018 v. a. Zugewanderte aus Deutschland (19,0\%), Italien (9,3\%), dem Vereinigten Königreich (6,6 \%), Portugal (5,8 \%), dem Kosovo (3,5\%), Spanien (3,3\%), Serbien (3,1\%), Frankreich $(3,0 \%)$, der Türkei (2,8 \%) und Österreich (2,7\%). Danach folgen die Zugewanderten mit den Staatsangehörigkeiten von Bosnien und Herzegowina (2,6 \%), den Niederlanden (2,5\%), Russland $(2,3 \%)$, Kroatien $(2,2 \%)$ und den USA (1,9\%). Darin zeichnet sich bereits ein etwas deutlicheres Bild über Zugs ständiger Wohnbevölkerung ohne Schweizer Pass ab, die im Jahr 2018 dort beheimatet ist. Zug hat neben den für die Schweiz klassischen Einwanderergruppen aus Italien, Spanien und Portugal sowie dem ehemaligen Jugoslawien, sehr viele Zuwanderinnen und Zuwanderer der sogenannten neuen Zuwanderung, allen voran aus Deutschland und dem Vereinigte Königreich vorzuweisen. Gleiches gilt für die Anteile aus den Nachbarländern Frankreich und Österreich, die noch unter den ersten zehn am stärksten vertretenen Nationalitäten in Zug zu finden sind. Etwas weiter entfernte Länder wie die Niederlande, Russland und 
die USA sind ebenfalls unter den ersten fünfzehn Ländern und können ebenfalls eher der Kategorie der neuen Zuwanderung in der Schweiz zugeordnet werden.

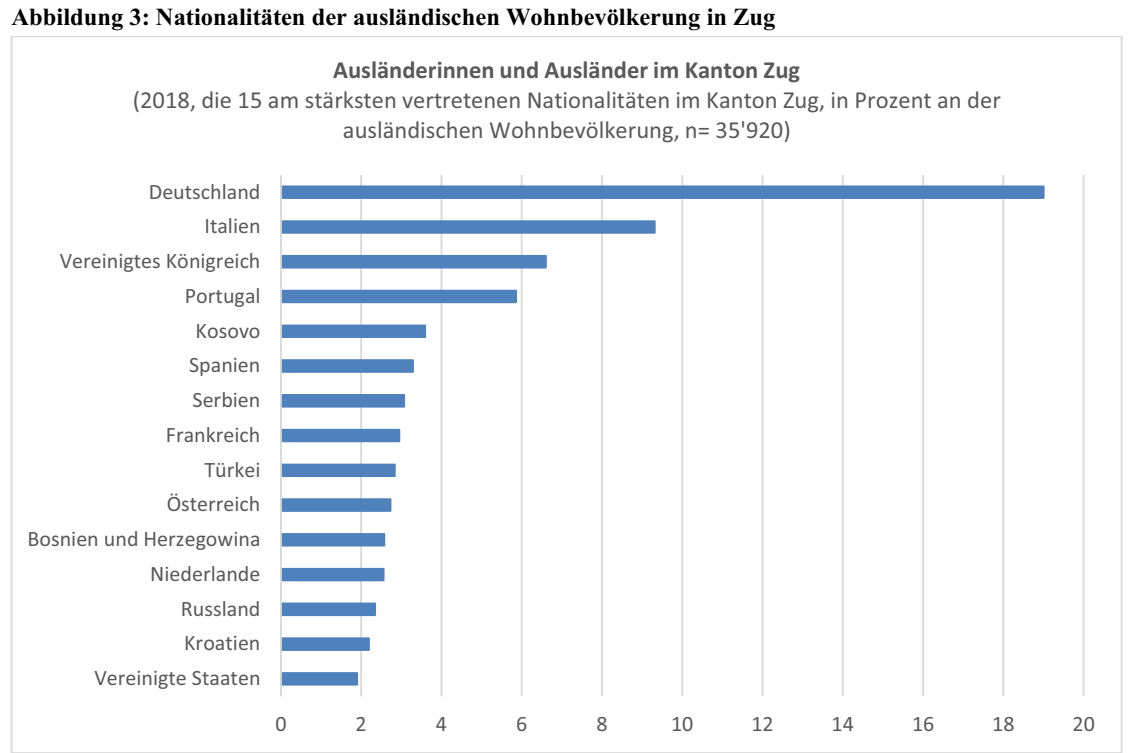

Quelle: Eigene Darstellung, Datengrundlage Bundesamt für Statistik, STATPOP, Datenblatt Nationalitäten im Kanton Zug 2006 bis 2018.

Ziehen wir zu den aktuellen Zahlen der fünfzehn am stärksten vertretenen Nationalitäten in Zug noch die Entwicklung der letzten zwölf Jahre hinzu, zeigt sich, dass sich die Zuwanderung je nach Nationalität unterschiedlich stark entwickelt hat. So kann in folgender Darstellung zunächst festgestellt werden, dass es v. a. die Gruppe der deutschen Zugewanderten in Zug ist, die bereits 2006 mit 18,7 Prozent den grössten Anteil der ausländischen Wohnbevölkerung im Kanton ausmachte. Im Jahre 2012 stieg dieser Anteil sogar noch auf 21,1 Prozent an, um sechs Jahre später auf 19,0 Prozent abzusinken. In absoluten Zahlen heisst das: Waren im Jahr 2006 noch 4'200 Personen mit deutschem Pass in Zug beheimatet, so waren es im Jahr 2012 bereits 6'223 Personen. Am Ende des Jahres 2018 lebten schliesslich 6'827 Menschen mit deutscher Staatsangehörigkeit im Kanton Zug. Insgesamt stieg die absolute Zahl der deutschen Zugewanderten zwar an, im Verhältnis zur Gesamtzahl aller Zugewanderten in Zug (die in den letzten zwölf Jahren von 22'496 (2006) über 29'455 (2012) auf insgesamt 35'920 (2018) Personen anstieg) ging sie jedoch wieder leicht zurück. Abbildung 4 zeigt aber noch eine weitere wichtige Entwicklung. Es wird deutlich, wie sich die Zuwanderung in den Kanton Zug über die letzten zwölf Jahren zugunsten von anderen Zuwanderungsgruppen weiter ausdifferenziert hat. So lässt sich erkennen, dass die Anteile der für die Schweiz als klassisch geltende Zuwanderungsländer wie Italien und Portugal eher zurückgehen bzw. sich gleichbleibend einpendeln; die Anteile der Zugewanderten aus Serbien, Bosnien und Herzegowina, Kroatien, Mazedonien und der Türkei 
gehen im Kanton Zug hingegen alle zurück. Zuwächse sind in erster Linie aus Ländern der sogenannten neuen Zuwanderung zu verzeichnen, darin v. a. aus dem Vereinigten Königreich (von 3,3 \% in 2006 auf 6,6 \% in 2018), gleiches gilt für zugewanderte Personen aus Frankreich (2006: 1,7 \%, 2018: 2,9\%) und Russland (2006: 0,8\%, 2018: 2,3 \%). Überwiegend konstant bleiben die Anteile der Zugewanderten aus den Niederlanden, Österreich und den USA im Kanton Zug.

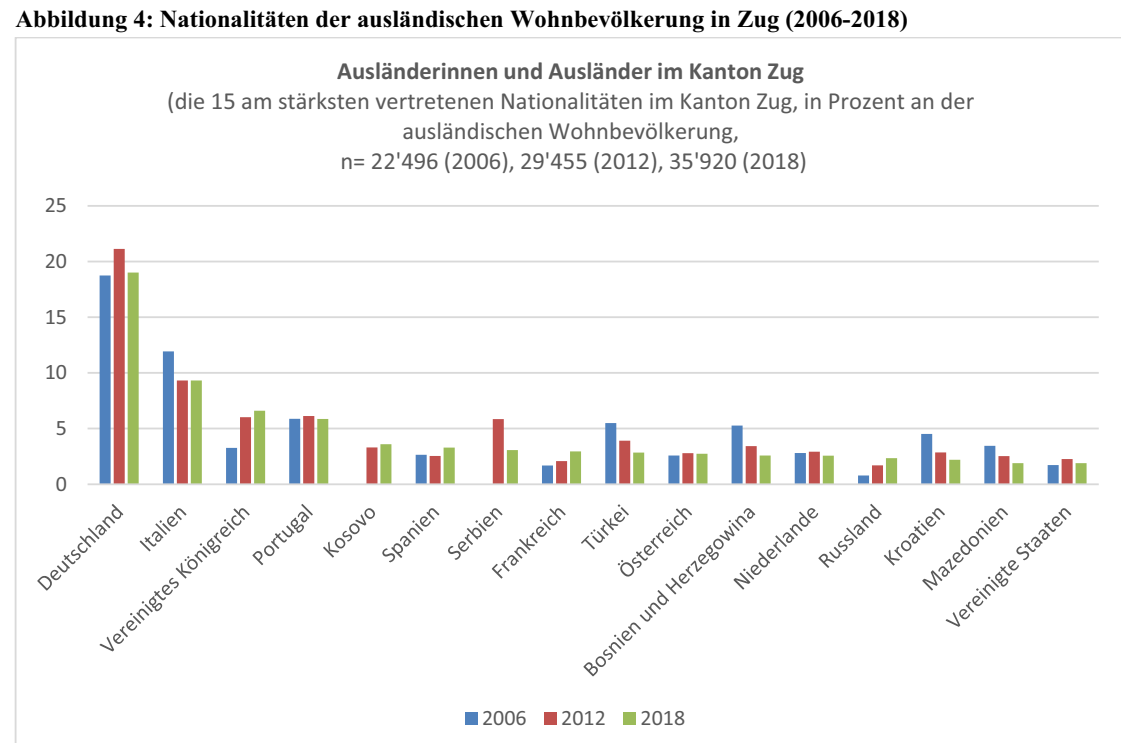

Quelle: Eigene Darstellung, Datengrundlage Bundesamt für Statistik, STATPOP, Datenblatt Nationalitäten im Kanton Zug 2006 bis 2018.

Anhand der dargestellten offiziellen Bevölkerungszahlen des Kantons Zug kann also deutlich gemacht werden, wie sich die Anteile der in Zug lebenden Ausländerinnen und Ausländer in den letzten zwölf Jahren in Richtung der sogenannten neuen Zuwanderung verändert bzw. ausdifferenziert haben. Um diesen Begriff hier noch einmal aufzugreifen: Nach der gängigen Definition charakterisiert sich diese neue Form v. a. durch eine starke Zunahme hochqualifizierter Ausländerinnen und Ausländer, die vornehmlich aus den nördlichen und westlichen Ländern einwandern, und durch einen Rückgang von Niedrigqualifizierten, die v. a. aus den südlichen Ländern in die Schweiz kommen. (vgl. z. B. Stutz et al. 2010: 7ff.) Obwohl sich eine solche Einteilung in alte und neue Zuwanderung gut eignet, die gegenwärtige Entwicklung zu beschreiben, kann sie ebenso zu vorschnellen Zuschreibungen führen, v. a. dann, wenn mit bestimmten Herkunftsregionen pauschal niedrige (dem ,globalen Süden“) oder hohe (dem „Norden“ und „Westen“) Qualifikationen verbunden werden. Selbstverständlich gibt es viele hochqualifizierte Menschen aus Regionen dieser Welt, die für gewöhnlich nicht sofort mit einer 
hohen Ausbildung in Verbindung gebracht werden. Um sich dem Phänomen der neuen Zuwanderung der Schweiz und damit insbesondere den Expatriates weiter statistisch anzunähern, lohnt noch ein Blick auf die Ausbildungsquoten der zugewanderten Menschen in der Schweiz. So hebt insbesondere Ganga Jey Aratnam (2012) in seiner Studie „Hochqualifizierte mit Migrationshintergrund" für die Schweiz auf Basis der Daten der Schweizerischen Arbeitskräfteerhebung (SAKE) hervor, dass sich in den wirtschaftlichen Grossregionen der Schweiz (Basel, Zürich, Genfer See) die Anteile der hochqualifizierten Zugewanderten in den letzten 15 Jahren erhöht hat. (ebd.: 141) „Hochqualifizierte“, so der Autor, „wandern bevorzugt in städtische Ballungsräume und deren Einzugsgebiete ein“. (ebd.: 139) Gleiches kann für den Kanton Zug angenommen werden (vgl. Stutz et al. 2010: 55), der zum Einzugsgebiet des Grossraums Zürich gehört.

Bei dem Versuch, sich dem Phänomen der Expatriates mit offiziellen Bevölkerungszahlen der Schweiz weiter zu nähern, kommt man spätestens an dieser Stelle an Grenzen. Weitere statistische Näherungen könnten zwar noch über Einkommen, Haushaltsgrösse, Aufenthaltsstatus und gegebenenfalls über die Wohnorte von Zugewanderten vorgenommen werden, wie es bspw. Wiener und Grossmann (2011) für ihre Studie über die Expatriates im Raum Basel getan haben. Doch selbst dann wäre nur eine gewisse Näherung an die Personengruppen der Expatriates möglich. Offizielle „Expatriate-Statistiken“ für die Schweiz und ihre Regionen existieren nicht, was vermutlich dem für gewöhnlich eher diffusen Begriffsverständnis von Expatriate geschuldet ist. Wie in dem bereits erwähnten Artikel von Marty (2010) schon deutlich wurde, ist es zudem nicht so einfach, einen typischen Expatriate zu charakterisieren. „Zu verschieden sind Sprachen, Kultur, Pläne und Lebensumstände““. (ebd.: 34) Nicht zuletzt könnte es auch an der Selbstzuschreibung vieler dieser Zugewanderter liegen, die nicht als Migrantin oder Migrant wahrgenommen werden möchten, sondern sich explizit als Expatriate verstehen. (Cattacin/Domenig 2012: 15; vgl. Duchêne-Lacroix 2006)

Die beschriebene Ausgangslage macht für mein Forschungsvorhaben vor allem zweierlei deutlich. Zum einen bewege ich mich in einem für die Schweiz zwar sehr aktuellen, aber noch wenig systematisch erforschten Bereich, welcher eine qualitative Forschungsanlage zwingend notwendig macht (vgl. Kap. 6). Zum anderen braucht es zu Beginn der Arbeit eine detaillierte Auseinandersetzung und Positionierung im Umgang mit dem zentralen Begriff Expatriate (vgl. Kap. 3).

\subsection{Erkenntnisinteresse und Forschungsfragen}

Wie einleitend beschrieben, nimmt die Studie ein Phänomen der sogenannten ,neuen Zuwanderung" (Stutz et al. 2010: 7ff.) der Schweiz in den Blick und untersucht das freiwillige Engagement von Expatriates im Kanton Zug. Im Besonderen richtet sich in meiner Arbeit der Fokus auf die Untersuchung der Ansprüche, Ressourcen und Beteiligungsmöglichen von Expatriates im zivilgesellschaftlichen Bereich. Die Studie analysiert, wo und in welcher Form sich Expatriates in Zug engagieren bzw. unter welchen Rahmenbedingungen dies geschieht. In der Studie steht daher das Alltagsleben von Expatriates in Zug im Mittelpunkt. Zugleich wird aber auch die Perspektive der zivilgesellschaftlichen Akteurinnen und Akteure des Gastlandes Schweiz 
untersucht, um so mögliche Anknüpfungs- resp. Erweiterungsformen für das zivilgesellschaftliche Engagement von Expatriates zu eruieren. D. h. neben der Perspektive der Expatriates werden zudem die Sichtweisen von in Zug etablierten Vereinen und Organisationen mit einbezogen. Die Studie prüft, ob bereits Erfahrungen mit Expatriates bestehen und welche kollektiven Umgangsweisen es in den Vereinen gibt.

Der Studie liegen daher folgende forschungsleitenden Fragen zugrunde:

- Wo und in welcher Form engagieren sich Expatriates im Kanton Zug?

- Welche Ansprüche und Ressourcen lassen sich bei Expatriates im Hinblick auf ihre zivilgesellschaftliche Beteiligung erkennen?

- Welche Faktoren wirken sich fördernd oder hemmend auf das zivilgesellschaftliche Engagement von Expatriates aus?

- Welche Erfahrungen haben etablierte Vereine und Organisationen in Zug bereits mit Expatriates gemacht?

- Wie schätzen etablierte Vereine und Organisationen die Ansprüche und Ressourcen von Expatriates ein?

- Welche Anpassungen sind bei Vereinen und Organisationen nötig, um die Ressourcen der Expatriates besser zu nutzen bzw. systematischer miteinzubeziehen?

Open Access Dieses Kapitel wird unter der Creative Commons Namensnennung 4.0 International Lizenz (http://creativecommons.org/licenses/by/4.0/deed.de) veröffentlicht, welche die Nutzung, Vervielfältigung, Bearbeitung, Verbreitung und Wiedergabe in jeglichem Medium und Format erlaubt, sofern Sie den/die ursprünglichen Autor(en) und die Quelle ordnungsgemäß nennen, einen Link zur Creative Commons Lizenz beifügen und angeben, ob Änderungen vorgenommen wurden.

Die in diesem Kapitel enthaltenen Bilder und sonstiges Drittmaterial unterliegen ebenfalls der genannten Creative Commons Lizenz, sofern sich aus der Abbildungslegende nichts anderes ergibt. Sofern das betreffende Material nicht unter der genannten Creative Commons Lizenz steht und die betreffende Handlung nicht nach gesetzlichen Vorschriften erlaubt ist, ist für die oben aufgeführten Weiterverwendungen des Materials die Einwilligung des jeweiligen Rechteinhabers einzuholen.

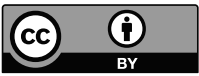

